Published in final edited form as:

Cancer Res. 2017 July 01; 77(13): 3655-3665. doi:10.1158/0008-5472.CAN-16-3199.

\title{
CXCL1 is critical for pre-metastatic niche formation and metastasis in colorectal cancer
}

\author{
Dingzhi Wang ${ }^{1, \#}$, Haiyan Sun ${ }^{2, \#}$, Jie Wei ${ }^{1}$, Bo Cen ${ }^{1}$, and Raymond N. DuBois ${ }^{1,3,{ }^{*}}$ \\ ${ }^{1}$ Department of Biochemistry and Molecular Biology, Medical University of South Carolina, \\ Charleston, SC 29425 \\ ${ }^{2}$ Laboratory for Inflammation and Cancer, Biodesign Institute of Arizona State University, Tempe, \\ AZ 85287
}

${ }^{3}$ Department of Research and Division of Gastroenterology, Mayo Clinic, Scottsdale, AZ 85259

\section{Abstract}

Emerging evidence suggests that the primary tumor influences the development of supportive metastatic microenvironments, referred to as pre-metastatic niches, in certain distant organs before arrival of metastatic cells. However, the mechanisms underlying the contributions of the primary tumor to pre-metastatic niche formation are not fully understood. Here we demonstrate that colorectal carcinoma cells secrete VEGF-A, which stimulates tumor-associated macrophages to produce CXCL1 in the primary tumor. Elevation of CXCL1 in pre-metastatic liver tissue recruited CXCR2-positive myeloid-derived suppressor cells (MDSC) to form a pre-metastatic niche that ultimately promoted liver metastases. Importantly, pre-metastatic liver-infiltrating MDSC induced tumor cell survival without involvement of innate or adaptive immune responses. Our study provides the first evidence that primary malignant cell-secreted VEGF-A stimulates tumorassociated macrophages to produce CXCL1, which recruits CXCR2-positive MDSC to form a premetastatic niche to promote liver metastases. Our findings not only shed light on how the tumor microenvironment contributes to pre-metastatic niche formation at distant sites, but they also provide comprehensive insights into how MDSC are recruited to other organs where they contribute to metastatic spread of disease. Moreover, our work also provides a rational for development of CXCR2 antagonists to inhibit or prevent metastatic spread of disease.

\section{Keywords}

CXCL1; pre-metastatic niches; colorectal cancer; myeloid-derived suppressor cells; VEGF

\section{Introduction}

Colorectal cancer (CRC) is the second-leading cause of cancer deaths and the third most common malignant neoplasm in the USA. Depending on the stage of disease at diagnosis,

\footnotetext{
"Correspondence to: Raymond N. DuBois, MD. Ph.D., 601 Clinical Science Building, 96 Jonathan Lucas Street, Suite 601, Charleston, SC 29425, Tel: 843-792-2842 and Fax: 843-792-2967, duboisrn@ musc.edu.

\#Equal contribution to the manuscript

Conflict of interest disclosure statement: All authors have no any conflict interests
} 
liver metastases occur in $20 \%$ to $70 \%$ of patients and lung metastases in $10 \%$ to $20 \%$ of patients. Unfortunately, distant metastases are the major cause of death for patients with advanced CRC because standard treatment options for widely metastatic CRC have not been as effective as needed, resulting in a very low 5-year survival rates. Clearly, understanding the molecular mechanisms responsible for CRC metastases will lead to novel strategies for treatment of advanced disease.

The metastatic spread of carcinoma cells is a complex process. The observations that no new mutations occur in the metastatic tumors when compared to primary colorectal carcinomas in the same patient (1) and that no new mutations were found in primary tumors of CRC patients with or without distant metastases (2) suggest that new mutations are not required to enable a tumor cells to leave the primary tumor and spread to secondary organs. Recent evidence suggests that tumor spread is facilitated by formation of supportive metastatic microenvironments, referred to as pre-metastatic niches, that develop prior to primary tumor cell dissemination (3). Pre-metastatic niches support colonization, survival, and growth of metastasizing tumor cells. However, the mechanisms responsible for formation of premetastatic niches are not well understood.

Primary tumor cells are thought to initiate pre-metastatic niche formation via secretion of pro-inflammatory cytokines, chemoattractants, and angiogenic factors that recruit and mobilize bone marrow-derived cells into future metastatic sites. For example, primary tumor-secreted VEGF or PIGF directly recruits bone marrow-derived VEGFR1 ${ }^{+}$ hematopoietic progenitor cells into future metastatic sites to develop pre-metastatic niches in mouse models of lung cancer and melanoma (4). Similarly, lysyl oxidase or CCL2 secreted from hypoxic mammary tumor cells induces pre-metastatic niche formation in lung via recruitment of bone marrow-derived cells and bone marrow-derived granulocytic myeloidderived suppressor cells (MDSCs), respectively $(5,6)$. Alternatively, primary tumor-secreted VEGF-A, TGF $\beta$, or TNFa induces A100A8/9 expression in resident cells of pre-metastatic lung, where A100A8/9 attracts MAC ${ }^{+}$-myeloid cells to form pre-metastatic niches (7). These previous studies suggest that primary tumor cell-secreted factors either directly recruit bone marrow-derived cells to pre-metastatic tissues or interact with resident cells of premetastatic organs to generate a pre-metastatic niche. However, it is not clear whether the microenvironment in the primary tumor also contributes to pre-metastatic niche formation.

CXCL1 levels are elevated in CRC and increased levels are associated with tumor size, advancing stage, depth of invasion, and patient survival $(8,9)$. Our previous work revealed that the CXCL1 receptor, CXCR2, is required for recruitment of granulocytic MDSCs (gMDSCs) into colonic mucosa and colitis-associated tumors from the circulatory system (10). Indeed, the levels of MDSCs in the blood and/or tumor tissue correlated with clinical cancer stage, metastatic tumor burden, or poor survival in patients with colon, esophageal, gastric, or pancreatic cancers (11-17). MDSCs have been shown to contribute to cancer immune evasion by suppressing effector T cell activation, proliferation, trafficking, viability, inhibition of natural killer cells (NKs), and activation/expansion of Treg cells (18). Our previous study also showed that MDSCs promoted inflammation-associated tumorigenesis by inhibiting $\mathrm{CD}^{+} \mathrm{T}$ cell cytotoxicity against tumor cells (10). Emerging evidence reveals that MDSCs accumulate in pre-metastatic sites $(5,19,20)$. However, the underlying 
mechanisms responsible for the recruitment of MDSCs into pre-metastatic organs and the contribution of MDSCs to colonization, survival, and outgrowth of metastasizing tumor cells remain elusive.

In this study, we investigate whether the molecular interactions of malignant cells with associated stromal cells in the primary tumor contribute to the formation of pre-metastatic niches and how these pre-metastatic niches facilitate metastasis.

\section{Materials and Methods}

\section{Cell culture and reagents}

HCT-116 and LS-174T cell lines were obtained from ATCC (Manassas, VA) in 2014. HCT-116, HCT-116/GFP-Luc, HCT116/vector, and HCT-116/shVEGF-A, LS-174T, LS-174T/GFP-Luc, LS-174T/vector, and LS-174T/shVEGF-A cells were cultured in McCoy's 5A medium with 10\% fetal bovine serum (FBS). All CRC cells are used between passages 2 to 5. HCoEpiC were obtained from ScienCell Research Laboratories (Carlsbad, CA) in 2014 and cultured in Colonic Epithelial Cell Medium (ScienCell Research Laboratories). HCoEpiC cells are used between passage 1 to 2 . All cell lines have been tested by MycoProbe Mycoplasma Detection Kit (R\&D) and also authenticated before the experiment according ATCC STR database. Primary carcinoma cells isolated from human CRC specimens were cultured in Chang medium with supplements (Irvine Scientific, Santa Ana, CA). For bone marrow-derived macrophages (BMDMs), bone marrow cells were flushed aseptically from the femurs of NSG mice and cultured in Falcon ${ }^{\mathrm{TM}}$ petri dishes (BD Biosciences, Cockeysville, MD) with DMEM medium supplemented with 10\% FBS and 10 $\mathrm{ng} / \mathrm{ml}$ of macrophage colony stimulating factor (M-CSF) for 6 days. After BMDMs were cultured in serum-free DMEM medium for $48 \mathrm{hr}$, the cells were treated with indicated dose of recombinant human VEGF-A (R\&D system, Minneapolis, MN) for $24 \mathrm{hr}$. The supernatants were collected and subjected to ELISA. The antibodies against CD45-PB, Ly6C-PE, and CD11c-PE-Cy7 were purchased from Biolegend (San Diego, CA), the antibodies against Ly6G-Alexa700 and F4/80-PE TexasRed were obtained from BD Biosciences, the antibody against CD11b-FITC was purchased from Miltenyi Biotec (Auburn, CA), the antibody against CXCR2-PE was purchased from R\&D systems, and the antibody against mouse CXCL1 was purchased from PeproTech (Rocky Hill, NJ). Murine rM-CSF was obtained from PeproTech.

\section{Animal experiments}

All animal experiments conformed to our animal protocols that were approved by the Institutional Animal Care and Use Committee at Arizona State University. NSG mice were obtained from Jackson Laboratory (Bar Harbor, Maine). For cecal injection, $5 \times 10^{4}$ of HCT-116-derived cells (HCT-116/GFP-Luc, HCT116/vector, and HCT-116/shVEGF-A), 2.5 $\times 10^{4}$ of LS-174T-derived cells (LS-174T/GFP-Luc, LS-174T/vector, and LS-174T/ shVEGF-A), or $2.5 \times 10^{5}$ of cancer cells derived from human primary CRC specimen were injected into the cecal wall of male NSG mice at age of 8 weeks old. After cecal injection, mice bearing HCT-116 tumors were sacrificed in 4 or 7 weeks for the experiments of premetastatic phase or metastatic stages. Similarly, mice bearing LS-174T or human primary 
carcinoma tumors were sacrificed in 7 or 11 weeks for the experiments of pre-metastatic phase or metastatic stages. $2 \times 10^{9}$ pfu of adeno-cre in $100 \mu \mathrm{L}$ PBS were administrated into the distal colon of WT or Apctlox/flox/LSL-Kras ${ }^{G 12 D}$ male mice aged at 3 months and the mice were sacrificed at aged 12 months. For CXCR2 antagonist (SB225002, Sigma-Aldrich) treatment, male NSG mice injected with HCT-116 cells were treated with SB225002 at 400 $\mu \mathrm{g} / 100 \mu \mathrm{L}$ PBS per mouse or vehicle by daily intraperitoneal injection for 4 or 7 weeks after injection. Similarly, male NSG mice injected with LS-174T cells were treated with same dosage of SB225002 for 7 or 11 weeks after cecal injection.

\section{Isolation of immunocytes from organs}

Liver was excised after perfusion of portal vein with cold-PBS and lung was excised after flushing with cold-PBS. Excised liver, lung, cecal tumor, and normal cecum were weighted, minced, and digested by digestion buffer $(3 \mathrm{mg} / \mathrm{ml}$ dispase II, $127.5 \mathrm{U} / \mathrm{ml}$ collagenase A, and $25 \mathrm{U} / \mathrm{ml}$ DNase I in HBSS) with 5\% FBS for $1 \mathrm{hr}$ at $37^{\circ} \mathrm{C}$ under slow rotation. Dispase II and DNase I were purchased from Roche, whereas collagenase A is obtained from Gibco. Discontinuous (44\% and 67\%) percoll (GE) separation method was used to enrich immunocytes. Whole blood was collected and bone marrow was isolated from the right ventricle and femurs. The RBCs in blood and bone marrow were lysed with RBC lysis buffer (eBioscience). $1 \times 10^{6}$ of immunocytes isolated from liver, lung, blood, bone marrow, cecal tumor, and normal cecum was subjected to the flow cytometry analyses.

\section{Flow cytometry analysis and sorting}

For multicolor flow cytometry immunotypic analyses, immunocyte cells were incubated with the appropriate combination of the following antibodies in staining buffer (BD Biosciences) at the following dilution: CD45-PB (1:200), CD11b-FITC (1:11), Ly6C-PE (1:80), Ly6G-Alexa700 (1:40), CD11c-PE-Cy7 (1:80), F4/80-PE TexasRed (1:80), and/or CXCR2-PE for $30 \mathrm{~min}$ on ice. After the cells were washed twice with $1 \mathrm{ml}$ of the labeling buffer, they were analyzed on a Gallios flow cytometer (Beckman Coulter). For analysis of CXCL1 expression on immune cells, immunocyte cells were stained with cell surface markers as described above. Then, the cells were fixed and permeabilized by using a Cytofix/Cytoperm kit (BD Biosciences) followed by intracellular cellular staining with primary rabbit anti-mouse CXCL1 antibody $(5 \mu \mathrm{g} / \mathrm{ml})$ and second anti-rabbit-IgG-APC or anti-rabbit-IgG-FITC (1:1000, Biolegend) in permeabilization buffer for $30 \mathrm{~min}$ on ice. After the cells were washed twice with $1 \mathrm{ml}$ of the permeabilization buffer, they were analyzed on a Gallios flow cytometer (Beckman Coulter). The flow cytometric profiles were analyzed by counting 20,000 events using Kaluza software program (Beckman Coulter). $\mathrm{CD} 11 \mathrm{~b}^{+}$Ly6 $\mathrm{G}^{\text {high }}$ cells from blood and liver were sorted by Gallios flow cytometer (Beckman Coulter).

\section{ELISA}

Total proteins were extracted from normal cecum, cecal tumors, livers, and lungs by homogenizing and subsequently sonicating in anti-protease buffer (50 mM HEPES, $150 \mathrm{mM}$ $\mathrm{NaCl}$, and $1 \mathrm{mM}$ EDTA) containing protease inhibitor cocktail tablets (Roche). The levels of human and mouse CXCL1, VEGF-A, and other factors in mouse tissues and cell-free 
supernatants were measured by using Quantikine ELISA kit (R\&D) according to the manufacturer's instructions.

\section{Lentivirus production and stable transfection}

HCT-116 and LS-174T cells were transduced with pLV-TH-GFP-Luc vector. VEGF-A shRNA and its vector were purchased from GE Dharmacon (Pittsburgh, PA). Lentivirus production and stable transfection were performed according to the manufacturer's instructions.

\section{Apoptosis assays}

The HCT-116 cells $\left(2.5 \times 10^{5} /\right.$ each well $)$ were plated in the lower chamber of 6 -transwell plates $(0.4 \mu \mathrm{M}$, Corning). After culture overnight, the cells were washed twice with PBS and then MDSCs were placed in the upper chamber at ratios (gMDSCs:HCT-116=0:1, 0.5:1, 1:1, $2: 1$, or $4: 1$ ) for 3 days culture. The percent of apoptotic tumor cells was determined by flow cytometry using TACS $^{\mathrm{TM}}$ Annexin V-FITC Apoptosis Detection Kit according to the manufacturer's instructions (R\&D System). The combination of Annexin V-FITC and propidium iodide allowed for the differentiation between early apoptotic cells (Annexin VFITC positive), late apoptotic cells (Annexin V-FITC and propidium iodide positive), necrotic cells (propidium iodide positive), and viable cells (both negative).

\section{Statistical analysis}

Each in vitro experiment was done at least 3 times and each in vivo experiment was conducted at least twice. Data are presented as mean \pm SEM. Comparisons among multiple groups were performed by factorial analysis of variance, followed by Bonferroni test. Comparisons between two groups were performed with Student's $t$-test or Mann-Whitney U test where appropriate. Fischer's exact test was used for categorical variables. $p<0.05$ was considered significant.

\section{RESULTS}

\section{Primary tumor induces an accumulation of MDSCs in pre-metastatic liver}

Since orthotopic mouse models of CRC allow us to distinguish human cancer cell-secreted factors from mouse host stromal cell-secreted factors, we were able to identify factors that directly attract bone marrow-derived cells into pre-metastatic organs were secreted from human malignant cells and/or from mouse host stromal cells. To precisely separate the premetastatic phase from metastatic stages in this model, human colorectal carcinoma HCT-116/GFP-Luc and LS-174T/GFP-Luc cells, which stably express GFP and luciferase, were injected into the cecal wall of NOD/scid/IL- $2 R \gamma^{-/-}$(NSG) mice. After injection, cecal tumor growth, liver metastasis, and lung metastasis were monitored by bioluminescence. For HCT-116 cells, cecal tumors formed in one week as judged by examining histological sections and luciferase activity. Since no luciferase activity in liver and lung was detected and no GFP cells were found in liver and lung at 4 weeks, all experiments for the "premetastatic" phase were performed within 4 weeks after injection. Similarly, all experiments with injection of LS-174T cells for the pre-metastatic phase were performed within 7 weeks after injection. 
We first evaluated whether the presence of a primary tumor affected immune cell profiles in pre-metastatic liver and lung of NSG mice. As shown in Fig. 1A-B, the presence of cecal tumors from HCT-116 or LS-174T cells induced accumulation of CD11b ${ }^{+}$Ly6 $6{ }^{\text {high }}$ CD11c ${ }^{-} \mathrm{F} 4 / 80^{-}$cells in pre-metastatic liver and lung as compared to normal cecal tissues injected with human colonic epithelial cells (HCoEpiC) as a control. NSG mice injected with HCoEpiC cells did not develop cecal tumors. Moreover, CD11 $b^{+}{ }^{+} y 6 \mathrm{G}^{\text {high }} \mathrm{CD} 11 \mathrm{c}^{-} \mathrm{F} 4 / 80^{-}$ cells isolated from pre-metastatic livers were able to inhibit the proliferation and IFN $\gamma$ production of activated $\mathrm{CD} 8^{+} \mathrm{T}$ cells in vitro, demonstrating that these cells are MDSCs (Supplementary Fig. S1A-B). Interestingly, MDSCs isolated from pre-metastatic liver inhibit $\mathrm{CD}^{+} \mathrm{T}$ cell activity more effectively than bone marrow-derived MDSCs (Supplementary Fig. S1A-B). Cecal tumors also induced infiltration of macrophages $\left(\mathrm{CD} 11 \mathrm{~b}^{+} \mathrm{F} 4 / 80^{+} \mathrm{Ly} 6 \mathrm{G}^{-} \mathrm{CD} 11 \mathrm{c}^{-}\right)$in only pre-metastatic livers (Fig. 1A-B). In contrast, the presence of cecal tumors did not affect accumulation of dendritic cells (CD11 $\left.\mathrm{c}^{+} \mathrm{Gr} 1^{-} \mathrm{F} 4 / 80^{-}\right)$ or neutrophils (CD11 $\mathrm{b}^{+} \mathrm{Ly} 6 \mathrm{G}^{+} \mathrm{CD} 11 \mathrm{c}^{-} \mathrm{F} 4 / 80^{-}$, but not $\mathrm{CD} 11 \mathrm{~b}^{+} \mathrm{Ly}_{6 \mathrm{G}}{ }^{\text {high }} \mathrm{CD} 11 \mathrm{c}^{-} \mathrm{F} 4 / 80^{-}$) (Fig. 1A-B and Supplementary Fig. S1C). RT-PCR analyses further confirmed that no luciferase gene was detected in liver or lung tissues, demonstrating that the presence of a cecal tumor alters immune cell profiles in pre-metastatic liver and lung. In mice, MDSCs are broadly defined as $\mathrm{CD} 11 \mathrm{~b}^{+} \mathrm{Gr} 1^{+}$cells that suppress the proliferation and the cytokine production of T cells. Mouse MDSCs are further divided into 2 subsets, granulocytic $\left(\mathrm{CD} 11 \mathrm{~b}^{+} \mathrm{Ly}_{6 \mathrm{G}}{ }^{\text {high }} \mathrm{Ly} 6 \mathrm{C}^{\text {low }}\right)$ and monocytic $\left(\mathrm{CD} 11 \mathrm{~b}^{+}\right.$Ly6C $\left.{ }^{+}\right)$MDSCs (21). However, gMDSCs isolated from livers of NSG don't express low levels of Ly6C. In most experimental tumor models, granulocytic MDSCs (gMDSCs) are more markedly expanded than monocytic MDSCs (mMDSCs) $(10,21)$. Indeed, 90\% of MDSCs expressed both CD11b and Ly6 $\mathrm{G}^{\text {high }}$ markers, whereas $10 \%$ of MDSCs expressed both CD11b and Ly6C but not Ly6G, especially in pre-metastatic liver (Supplementary Fig. S1C-D). Furthermore, human primary colon carcinoma cell-derived cecal tumors only induced an infiltration of gMDSCs in pre-metastatic liver and lung (Fig. 1C). We further examined whether colonic tumors also altered immune cell profiles in pre-metastatic liver and lung in a mouse model of spontaneous CRC, $A \mathrm{pc}^{-/-} / \mathrm{Kras}^{G 12 D}$ mice. Indeed, colonic tumors only promoted an accumulation of gMDSCs in pre-metastatic liver of $\mathrm{Apc}^{-/-} / \mathrm{KraS}^{G 12 D}$ mice as compared to WT mice (Fig. 1D). Since we only found gMDSC accumulation in pre-metastatic liver in the four animal models we tested, we concluded that primary tumors preferentially induce an infiltration of gMDSCs into pre-metastatic liver.

\section{The CXCL1-CXCR2 axis is required for recruitment of MDSCs into pre-metastatic liver and liver metastasis}

Since CXCR2 has been shown to be required for homing of MDSCs into colonic mucosa and tumors from the circulatory system (10), we postulated that CXCR2 ligands such as CXCL1, CXCL2, and/or CXCL5 are able to recruit circulatory MDSCs into local organs via CXCR2. Indeed, HCT-116, LS-174T, and human primary colon carcinoma cells secreted higher levels of CXCL1 than HCoEpiC (Fig. 2A). Unexpectedly, human CXCL1 was not detected in pre-metastatic liver and lung although the levels of both human and mouse CXCL1 were elevated in cecal tumors as compared to normal cecal tissues injected with $\mathrm{HCoEpiC}$ as a control (Fig. 2B), demonstrating that human CXCL1 secreted from cancer cells is not responsible for recruitment of gMDSCs. In contrast, mouse CXCL1 was elevated 
in pre-metastatic liver (Fig. 2C) and blood (Fig. 2D) compared to levels observed in controls. Moreover, mouse CXCL2 and CXCL5 were not detected in pre-metastatic liver. To exclude the possibility that other factors secreted from human colorectal carcinoma cells might directly attract gMDSCs, an array of human chemokines, cytokines, and growth factors such as CXCL2, CXCL3, CXCL5, CXCL8 (IL-8), CXCL9, CXCL10, CXCL11, CCL2, CCL3, CCL4, CCL5, IL-6, INF $\gamma$, and VEGF were examined in pre-metastatic liver and lung tissue. None of these factors were detected in pre-metastatic liver. These results demonstrate that primary tumor cell-secreted factors do not directly attract gMDSCs into pre-metastatic liver. Moreover, mouse CXCL1 was also increased in colonic tumors and premetastatic livers in $\mathrm{Apc}^{-/-} / \mathrm{Kras}^{G 12 D}$ mice (Fig. 2E). Collectively, these results indicate that mouse CXCL1 secreted from primary tumor stromal cells is responsible for attracting circulatory gMDSCs into pre-metastatic liver in our models.

We further determined whether the CXCL1-CXCR2 axis is required for recruitment of gMDSCs into pre-metastatic liver. Since CXCR2 is mainly expressed on $\mathrm{Ly}_{6 \mathrm{G}}{ }^{+}$cells, including neutrophils and MDSCs, in mice (22), we first confirmed whether gMDSCs express CXCR2 in our model. Almost all circulatory gMDSCs expressed CXCR2 on their cell surface and more than $80 \%$ of bone marrow gMDSCs expressed CXCR2 regardless of the presence or absence of cecal tumors (Fig. 2F). In contrast, almost all circulatory mMDSCs did not express CXCR2 regardless of the presence or absence of cecal tumors (Supplementary Fig. S1E). In addition, cecal tumors derived from HCT-116 and LS-174T cells increased gMDSC populations in blood but not in bone marrow (Supplementary Fig. S2A). Since our previous study demonstrated that $C x c r 2$-deficient gMDSCS have lost their ability to migrate to colonic mucosa and tumors (10), we postulated that inhibition of CXCR2 signaling attenuated primary tumor-induced infiltration of gMDSCs into premetastatic liver and inhibited liver metastasis. Indeed, CXCL1 attracted gMDSCs isolated from blood of NSG mice, but did not affect the expansion of gMDSCs isolated from the premetastatic livers (Supplementary Fig. S2B-C). Importantly, a CXCR2 antagonist (SB225002) inhibited CXCL1-induced chemotaxis (Supplementary Fig. S2D). These results demonstrate that CXCR2 is required for CXCL1 induction of MDSC chemotaxis. Moreover, treatment of mice with SB225002 significantly inhibited primary tumor-induced infiltration of gMDSCs in pre-metastatic livers (Fig. 2G) and reduced overall burden of metastatic disease in the liver (Fig. 2H). In addition, the CXCR2 antagonist significantly decreased infiltration of gMDSCs into pre-metastatic livers without affecting other immune cells such as dendritic cells (DCs) and macrophages (Fig. 2G), demonstrating that the CXCR2 antagonist specifically targets gMDSCs. Importantly, treatment with the CXCR2 antagonist resulted in accumulation of gMDSCs in the circulatory system (Supplementary Fig. S2E), demonstrating that inhibition of CXCR2 by its antagonists blocks the recruitment of gMDSCs into pre-metastatic livers from the circulatory system. Interestingly, the CXCR2 antagonist did not affect cecal tumor growth in both the pre-metastatic stage (Supplementary Fig. S2F, left panel) and metastatic stage (Supplementary Fig. S2F, right panel). Similarly, the CXCR2 antagonist also inhibited primary tumor-induced infiltration of gMDSCs in premetastatic livers (Supplementary Fig. S2 $\underline{G}$ ) and reduced liver metastatic tumor burden (Supplementary Fig. S2H) in mice injected with LS-174T cells without affecting cecal tumor growth (Supplementary Fig. S2I). Collectively, these results demonstrate that 
elevation of mouse CXCL1 in pre-metastatic liver recruits CXCR2-expressing gMDSCs from the circulatory system into pre-metastatic liver, which in turn promotes liver metastasis.

\section{VEGF is required for induction of CXCL1 and infiltration of MDSC in pre-metastatic liver as well as promotion of liver metastasis}

CXCL1 has been shown to be upregulated by pro-inflammatory cytokines TNFa and IL-1 $\beta$ or by bacterial infection in colorectal carcinoma cells (23). However, the levels of TNFa were undetectable in both LS-174T and HCT-116 cell culture supernatant, whereas IL-1 $\beta$ levels were undetectable in LS-174T cells and cecal tumors derived from HCT-116 cells. These results demonstrated that TNFa and IL- $1 \beta$ were not likely to be tumor cell-secreted factors that upregulate CXCL1 expression in our models. In contrast, VEGF-A levels were significantly higher in cell culture supernatants of LS-174T, HCT-116, and primary colon carcinoma cells than HCoEpiC cells (Fig. 3A). Both human and/or mouse VEGF-A were also elevated in cecal tumors of NSG mice (Fig. 3B) and colon tumors found in $\mathrm{Apc}^{-/-1}$ Kras ${ }^{G 12 D}$ mice (Fig. 3C). In contrast, human VEGF-B, -C, and -D were not detectable in cecal tumors. However, primary tumors did not induce mouse VEGF-A expression in premetastatic livers of both NSG mice and $A \mathrm{pc}^{-/-} / \mathrm{Kras}^{G 12 D}$ mice (Fig. 3D). As mentioned earlier, human VEGF is not detectable in pre-metastatic livers of NSG mice. These results demonstrate that human VEGF-A secreted from human cancer cells and mouse VEGF-A secreted from tumor stromal cells are not responsible for recruitment of gMDSCs. Since VEGF has been shown to induce CXCL1 expression in human lung carcinoma epithelial cells and endothelial cells $(24,25)$, we first examined whether cancer cell-secreted VEGF regulated CXCL1 expression. Knockdown of VEFG-A in HCT-116 and LS-174T cells reduced the levels of VEGF-A and CXCL1 in supernatants (Fig. 4A). Similarly, the levels of human VEGF, human CXCL1, and mouse CXCL1 were significantly reduced in cecal tumors derived from HCT-116/shVEGF-A and LS-174T/shVEGF-A cells as compared to vector cells (Fig. 4B). Importantly, knockdown of VEFG-A in HCT-116 and LS-174T cells significantly decreased mouse CXCL1 levels and gMDSC numbers in pre-metastatic livers as compared to controls (Fig. 4C-D). Finally, mice bearing cecal tumors derived from VEGF knockdown cells developed fewer liver metastatic lesions than that seen from vector cells (Fig. 4E-F). In contrast, knockdown of VEFG-A did not affect cecal tumor growth during the pre-metastatic stage (Supplementary Fig. S3A) and metastatic stage (Supplementary Fig. S3B). These results demonstrate that human VEGF-A secreted from primary tumor cells is required for induction of mouse CXCL1 in primary tumors and premetastatic liver as well as recruitment of gMDSCs into pre-metastatic liver and liver metastasis.

\section{Tumor cell-secreted VEGF stimulates tumor-associated macrophages to produce CXCL1}

Since human VEGF is not detectable in pre-metastatic liver, human VEGF induction of mouse CXCL1 must occur in cecal tumors. To determine which primary tumor associated stromal cells express CXCL1, flow cytometry analyses were performed. Cecal tumorassociated fibroblasts and endothelial cells did not express CXCL1. We further analyzed immune cell profiles in cecal tumor specimens and found that the majority of immune cells were macrophages following injection of HCT-116 cells (Fig. 5A), whereas the majority of 
immune cells were comprised of macrophages and MDSCs following injection of LS-174T cells (Fig. 5B). Mouse CXCL1 was mainly expressed in tumor-associated macrophages (TAMs) (Fig. 5C-D). Knockdown of VEGF-A in HCT-116 or LS-174T cells significantly reduced CXCL1-expressing macrophages in cecal tumors (Fig. 5E-F), indicating that VEGF-A secreted from tumor cells stimulates TAMs to produce CXCL1. In addition, TAMs mainly expressed VEGFR1 in the cecal tumors derived from HCT-116 cells (Supplementary Fig. S3C). We further examined whether VEGF-A is able to directly induce CXCL1 expression in macrophages. Treatment of mouse bone marrow-derived macrophages with human VEGF-A resulted in induction of CXCL1 expression (Fig. 5G), demonstrating that human VEGF-A can directly stimulate mouse macrophages to produce mouse CXCL1. Collectively, these results demonstrate that VEGF-A secreted from primary tumor cells stimulates primary tumor-infiltrating macrophages to produce CXCL1.

\section{MDSCs in pre-metastatic liver promote cancer cell survival}

Since NSG mice lack mature T cells, B cells, and functional NK cells, we questioned how MDSCs in pre-metastatic liver enhanced liver metastatic tumor formation and growth without involvement of innate and adaptive immune responses. To address this question, we determined whether MDSCs promote cancer cell survival by acting directly on cancer cells. Indeed, gMDSCs isolated from pre-metastatic livers of NSG mice bearing HCT-116 cecal tumors (panel A) or LS-174T cecal tumors (panel B) inhibited HCT-116 cell apoptosis induced by serum deprivation in cell culture without the cell-cell interaction of malignant cells and gMDSCs (Fig. 6). The gMDSCs enhancement of tumor cell survival was ratiodependent. Interestingly, bone marrow-derived gMDSCs from NSG mice were not able to promote cancer cell survival (Fig. 6), indicating that only MDSCs located in the premetastatic niche were able to promote cancer cell survival. These results reveal that liverinfiltrating gMDSCs-secreted factors are able to promote cancer cell survival without involvement of innate and adaptive immune responses. Taken together, these results demonstrate that primary tumor cell-secreted VEGF-A stimulates primary tumor-infiltrating macrophages to produce CXCL1 that recruits CXCR2-expressing MDSCs from the circulatory system into pre-metastatic liver. These MDSCs then promote metastatic tumor formation and growth via modulation of cancer cell survival (Fig. 7).

\section{DISCUSSION}

A growing body evidence demonstrates that pre-metastatic niches are critical for facilitating homing, survival, and growth of circulating tumor cells in secondary organs. However, the cellular and molecular mechanisms by which primary tumor cells influence the formation of pre-metastatic niches in secondary organs are not well understood. Our study not only reveals a novel mechanism by which the primary tumor facilitates pre-metastatic niche formation that promotes metastasis, but also may offer CXCR2 as a new target in treating or preventing advancement of CRC disease.

Previous studies have shown that primary tumor cell-secreted factors either directly recruit BMDCs to pre-metastatic lesions or interact with resident cells in the pre-metastatic sites to generate pre-metastatic niches. However, our observation that primary malignant cells- 
secreted factors were undetectable in pre-metastatic liver in our mouse model of CRC liver metastasis indicates that these factors could not directly recruit MDSCs to pre-metastatic lesions or interact with resident cells in the pre-metastatic sites to generate a pre-metastatic niche. Moreover, we provide the first evidence that malignant cell-secreted VEGF-A stimulates TAMs to produce CXCL1 that directly recruits circulatory MDSCs into premetastatic liver, which in turn promotes liver metastasis (Fig. 7). Our results demonstrate for the first time that primary tumor stromal cells contribute to the formation of pre-metastatic niches.

TAMs are recognized as a poor prognostic sign in various tumors, including colorectal cancer $(26,27)$. Multiple lines of evidence indicate that TAMs promote cancer progression and metastasis through supporting tumor-associated angiogenesis, enhancing tumor cell migration, invasion, and intravasation, and suppressing immuno-surveillance (28). For example, VEGF-A secreted from TAMs located in a primary tumor induces angiogenesis by binding to VEGFR1 and VEGFR2 on vascular endothelial cells (29). In addition, TAMs suppress $\mathrm{CD}^{+} \mathrm{T}$ cell cytotoxic activity by either expressing immune checkpoint receptor ligands such as PD-L1 and B7-H4 or recruiting Treg cells (30,31). However, it was unknown whether TAMs in primary tumor play a role in the formation of pre-metastatic niches. Our data reveal that CXCL1 secreted from TAMs is required for recruitment of MDSCs into premetastatic liver to form pre-metastatic niches. Although VEGFR1 and VEGFR2 are mainly expressed in vascular endothelial cells, VEGFR1 is also expressed in hematopoietic, monocytes/macrophage, and smooth muscle cells (32). For example, bone marrow-derived VEGFR $1^{+}$hematopoietic progenitor cells are required for pre-metastatic niche formation (4). Our data showed that TAMs expressed higher levels of VEGFR1 than macrophages in normal cecum, indicating that VEGF-A induces CXCL1 expression in TAMs via VEGFR1. Although VEGF-A has been shown to induce CXCL1 via PKD2 in endothelial cells (25) and to induce CXCL1 via JNK and PI3K pathways in human lung carcinoma epithelial cells (24), further research is needed to determine molecular mechanisms underlying VEFG-A induction of CXCL1 in tumor-associated macrophages. Unexpectedly, knockdown of VEGF-A in cancer cells did not affect primary tumor growth although VEGF-A knockdown indeed inhibited liver metastasis. Previous results are consistent with our observation. Ablation of macrophages by deletion of colony stimulating factor 1 (CSF1) significantly attenuates lung metastasis without affecting primary tumor growth in the PyMT model of breast cancer (33). Similarly, deletion of SRC1 reduces CSF-1 production in tumor cells and TAM numbers in primary tumor, which inhibits lung metastasis without affecting PyMT primary tumor formation (34). These studies indicate that TAMs are required for metastasis, but may not be involved in primary tumor growth.

It has been well established that CXCL1 promotes tumor growth via induction of angiogenesis. Recent evidence demonstrated that CXCL1 enhanced tumor growth via recruitment of neutrophils into the tumor microenvironment. For example, tumor cellsecreted CXCL1 promoted lung cancer growth via recruitment of neutrophils into tumor tissue in vivo (35). TNFa stimulates mesenchymal stromal cells to produce CXCR2 ligands that recruit $\mathrm{CXCR} 2^{+}$neutrophils into primary tumors in a mouse model of breast cancer (36). Infiltration of $\mathrm{CXCR} 2^{+}$neutrophils into primary tumor promotes lung metastasis. Inhibition of CXCR2 suppresses metastases by targeting Ly6G+ cells in mouse models of 
pancreatic cancer (37). Moreover, our group and others have demonstrated that the CXCR2 ligands-CXCR2 axis is required for infiltration of MDSCs into colonic mucosa and tumors $(10,38)$. In this study, we extended the scope of our research to reveal that this axis is also essential for formation of pre-metastatic niches and liver metastasis.

It is well established that CXCL1 can directly recruit circulatory CXCR2-expressing neutrophils and MDSCs into inflammatory sites and tumor tissues. Since CXCL1 has also been shown to indirectly mobilize hematopoietic stem cells (HSC) and hematopoietic progenitor cells (HPC) into the peripheral blood from bone marrow via MMP9 release from neutrophils $(39,40)$, CXCL1 may indirectly mobilize HSC and HPC into pre-metastatic liver, in which they differentiate into monocytes or granulocytes such as neutrophils and MDSCs. In addition, it remains unclear mechanistically how CXCL1 accumulated in pre-metastatic liver at higher concentrations than in the systemic circulation if CXCL1 produced in cecal tumor diffuses to pre-metastatic liver tissue via the circulatory system. It's possible that VEGF produced by the primary cecal tumor directs tumor-associated macrophages to localize in pre-metastatic liver and produce CXCL1. Further studies are needed to investigate the precise mechanisms regulating CXCL1 levels and CXCL1-induced accumulation of MDSCs in pre-metastatic liver.

Although substantial evidence demonstrates that primary tumors induce accumulation of bone marrow-derived immature myeloid cells in secondary organs before arrival of metastatic tumor cells, the functions of these cells in the pre-metastatic niche have been poorly characterized. One study showed that accumulation of MDSCs inversely correlated with suppression of NK cell cytotoxicity and maturity in pre-metastatic lung (5). However, the immunosuppressive function of MDSCs in pre-metastatic niches has not been investigated. One possible reason could be that $\mathrm{T}$ cells have not been directly linked to the pre-metastatic niche formation. Thus, the central question is how MDSCs in pre-metastatic niches promote metastatic tumor cell colonization, survival, and proliferation without involvement of NK and T cells. One study reported that MDSCs in pre-metastatic lung tissue might enhance mammary tumor cell extravasation by remodeling blood vessels via MMP9 (19). Moreover, emerging evidence notes that MDSCs enhance cancer stem cell formation and protects proliferating tumor cells from senescence by directly targeting these cells $(41,42)$. Here we provide the first evidence showing that MDSCs in pre-metastatic liver promote metastasis via directly enhance metastatic tumor cell survival. This finding reveals a novel function of MDSCs in promotion of cancer cell survival without involvement of innate and adaptive immune responses.

In summary, VEGF-A secreted by primary tumor cells stimulates primary tumor-associated macrophages to produce CXCL1 that recruits CXCR2-positive MDSCs to form premetastatic niches promoting liver metastases. MDSCs in pre-metastatic niches promote cancer cell survival by directly targeting cancer cells. These findings not only shed light on how primary tumor microenvironment contributes to pre-metastatic niche formation at distant sites, but also provide comprehensive insights into how MDSCs are recruited to secondary organs and how MDSCs contribute to metastasis. 


\section{Supplementary Material}

Refer to Web version on PubMed Central for supplementary material.

\section{Acknowledgments}

Financial support: DW, HS, and RND (NIH R01 DK047297 to R.N. DuBois, NCI R01 CA184820 to R.N. DuBois, NCI P01 CA077839 to R.N. DuBois), JW (NIH R01 DK047297 to R.N. DuBois, NCI R01 CA184820 to R.N. DuBois), BC (NCI P01 CA077839 to R.N. DuBois)

We thank the National Colorectal Cancer Research Alliance (NCCRA) for its generous support (R.N.D.). DW and RND conceived the project; HS, JW, and BC performed the experiments; DW analyzed the data and wrote the manuscript.

\section{References}

1. Jones S, Chen WD, Parmigiani G, Diehl F, Beerenwinkel N, Antal T, et al. Comparative lesion sequencing provides insights into tumor evolution. Proc Natl Acad Sci U S A. 2008; 105:4283-8. [PubMed: 18337506]

2. Mlecnik B, Bindea G, Kirilovsky A, Angell HK, Obenauf AC, Tosolini M, et al. The tumor microenvironment and Immunoscore are critical determinants of dissemination to distant metastasis. Science translational medicine. 2016; 8:327ra26.

3. Psaila B, Lyden D. The metastatic niche: adapting the foreign soil. Nat Rev Cancer. 2009; 9:285-93. [PubMed: 19308068]

4. Kaplan RN, Riba RD, Zacharoulis S, Bramley AH, Vincent L, Costa C, et al. VEGFR1-positive haematopoietic bone marrow progenitors initiate the pre-metastatic niche. Nature. 2005; 438:820-7. [PubMed: 16341007]

5. Sceneay J, Chow MT, Chen A, Halse HM, Wong CS, Andrews DM, et al. Primary tumor hypoxia recruits $\mathrm{CD} 11 \mathrm{~b}+/ \mathrm{Ly} 6 \mathrm{Cmed} / \mathrm{Ly} 6 \mathrm{G}+$ immune suppressor cells and compromises NK cell cytotoxicity in the premetastatic niche. Cancer Res. 2012; 72:3906-11. [PubMed: 22751463]

6. Erler JT, Bennewith KL, Cox TR, Lang G, Bird D, Koong A, et al. Hypoxia-induced lysyl oxidase is a critical mediator of bone marrow cell recruitment to form the premetastatic niche. Cancer Cell. 2009; 15:35-44. [PubMed: 19111879]

7. Hiratsuka S, Watanabe A, Aburatani H, Maru Y. Tumour-mediated upregulation of chemoattractants and recruitment of myeloid cells predetermines lung metastasis. Nature cell biology. 2006; 8:136975. [PubMed: 17128264]

8. Ogata H, Sekikawa A, Yamagishi H, Ichikawa K, Tomita S, Imura J, et al. GROalpha promotes invasion of colorectal cancer cells. Oncol Rep. 2010; 24:1479-86. [PubMed: 21042742]

9. Wang D, Dubois RN, Richmond A. The role of chemokines in intestinal inflammation and cancer. Curr Opin Pharmacol. 2009; 9:688-96. [PubMed: 19734090]

10. Katoh H, Wang D, Daikoku T, Sun H, Dey SK, Dubois RN. CXCR2-expressing myeloid-derived suppressor cells are essential to promote colitis-associated tumorigenesis. Cancer Cell. 2013; 24:631-44. [PubMed: 24229710]

11. Mandruzzato S, Solito S, Falisi E, Francescato S, Chiarion-Sileni V, Mocellin S, et al. IL4Ralpha+ myeloid-derived suppressor cell expansion in cancer patients. J Immunol. 2009; 182:6562-8. [PubMed: 19414811]

12. Diaz-Montero CM, Salem ML, Nishimura MI, Garrett-Mayer E, Cole DJ, Montero AJ. Increased circulating myeloid-derived suppressor cells correlate with clinical cancer stage, metastatic tumor burden, and doxorubicin-cyclophosphamide chemotherapy. Cancer Immunol Immunother. 2009; 58:49-59. [PubMed: 18446337]

13. Gabitass RF, Annels NE, Stocken DD, Pandha HA, Middleton GW. Elevated myeloid-derived suppressor cells in pancreatic, esophageal and gastric cancer are an independent prognostic factor and are associated with significant elevation of the Th2 cytokine interleukin-13. Cancer Immunol Immunother. 2011; 60:1419-30. [PubMed: 21644036] 
14. Duffy A, Zhao F, Haile L, Gamrekelashvili J, Fioravanti S, Ma C, et al. Comparative analysis of monocytic and granulocytic myeloid-derived suppressor cell subsets in patients with gastrointestinal malignancies. Cancer Immunol Immunother. 2013; 62:299-307. [PubMed: 23011590]

15. Sun HL, Zhou X, Xue YF, Wang K, Shen YF, Mao JJ, et al. Increased frequency and clinical significance of myeloid-derived suppressor cells in human colorectal carcinoma. World journal of gastroenterology : WJG. 2012; 18:3303-9. [PubMed: 22783056]

16. Zhang B, Wang Z, Wu L, Zhang M, Li W, Ding J, et al. Circulating and tumor-infiltrating myeloidderived suppressor cells in patients with colorectal carcinoma. PLOS One. 2013; 8:e57114. [PubMed: 23437326]

17. Wang L, Chang EW, Wong SC, Ong SM, Chong DQ, Ling KL. Increased myeloid-derived suppressor cells in gastric cancer correlate with cancer stage and plasma S100A8/A9 proinflammatory proteins. J Immunol. 2013; 190:794-804. [PubMed: 23248262]

18. Gabrilovich DI, Ostrand-Rosenberg S, Bronte V. Coordinated regulation of myeloid cells by tumours. Nat Rev Immunol. 2012; 12:253-68. [PubMed: 22437938]

19. Yan HH, Pickup M, Pang Y, Gorska AE, Li Z, Chytil A, et al. Gr-1+CD11b+ myeloid cells tip the balance of immune protection to tumor promotion in the premetastatic lung. Cancer Res. 2010; 70:6139-49. [PubMed: 20631080]

20. Gao D, Joshi N, Choi H, Ryu S, Hahn M, Catena R, et al. Myeloid progenitor cells in the premetastatic lung promote metastases by inducing mesenchymal to epithelial transition. Cancer Res. 2012; 72:1384-94. [PubMed: 22282653]

21. Youn JI, Nagaraj S, Collazo M, Gabrilovich DI. Subsets of myeloid-derived suppressor cells in tumor-bearing mice. J Immunol. 2008; 181:5791-802. [PubMed: 18832739]

22. Cacalano G, Lee J, Kikly K, Ryan AM, Pitts-Meek S, Hultgren B, et al. Neutrophil and B cell expansion in mice that lack the murine IL-8 receptor homolog. Science. 1994; 265:682-4. [PubMed: 8036519]

23. Verbeke H, Struyf S, Laureys G, Van Damme J. The expression and role of CXC chemokines in colorectal cancer. Cytokine \& Growth Factor Reviews. 2011; 22:345-58. [PubMed: 22000992]

24. Lo HM, Shieh JM, Chen CL, Tsou CJ, Wu WB. Vascular Endothelial Growth Factor Induces CXCL1 Chemokine Release via JNK and PI-3K-Dependent Pathways in Human Lung Carcinoma Epithelial Cells. International journal of molecular sciences. 2013; 14:10090-106. [PubMed: 23665907]

25. Hao Q, Wang L, Tang H. Vascular endothelial growth factor induces protein kinase D-dependent production of proinflammatory cytokines in endothelial cells. American journal of physiology Cell physiology. 2009; 296:C821-7. [PubMed: 19176759]

26. Qian BZ, Pollard JW. Macrophage diversity enhances tumor progression and metastasis. Cell. 2010; 141:39-51. [PubMed: 20371344]

27. Bacman D, Merkel S, Croner R, Papadopoulos T, Brueckl W, Dimmler A. TGF-beta receptor 2 downregulation in tumour-associated stroma worsens prognosis and high-grade tumours show more tumour-associated macrophages and lower TGF-beta1 expression in colon carcinoma: a retrospective study. BMC Cancer. 2007; 7:156. [PubMed: 17692120]

28. Kitamura T, Qian BZ, Pollard JW. Immune cell promotion of metastasis. Nat Rev Immunol. 2015; 15:73-86. [PubMed: 25614318]

29. Lin EY, Pollard JW. Tumor-associated macrophages press the angiogenic switch in breast cancer. Cancer Res. 2007; 67:5064-6. [PubMed: 17545580]

30. Kuang DM, Zhao Q, Peng C, Xu J, Zhang JP, Wu C, et al. Activated monocytes in peritumoral stroma of hepatocellular carcinoma foster immune privilege and disease progression through PDL1. J Exp Med. 2009; 206:1327-37. [PubMed: 19451266]

31. Kryczek I, Zou L, Rodriguez P, Zhu G, Wei S, Mottram P, et al. B7-H4 expression identifies a novel suppressive macrophage population in human ovarian carcinoma. J Exp Med. 2006; 203:871-81. [PubMed: 16606666]

32. Autiero M, Luttun A, Tjwa M, Carmeliet P. Placental growth factor and its receptor, vascular endothelial growth factor receptor-1: novel targets for stimulation of ischemic tissue 
revascularization and inhibition of angiogenic and inflammatory disorders. Journal of thrombosis and haemostasis : JTH. 2003; 1:1356-70. [PubMed: 12871269]

33. Lin EY, Nguyen AV, Russell RG, Pollard JW. Colony-stimulating factor 1 promotes progression of mammary tumors to malignancy. J Exp Med. 2001; 193:727-40. [PubMed: 11257139]

34. Wang S, Yuan Y, Liao L, Kuang SQ, Tien JC, O'Malley BW, et al. Disruption of the SRC-1 gene in mice suppresses breast cancer metastasis without affecting primary tumor formation. Proc Natl Acad Sci U S A. 2009; 106:151-6. [PubMed: 19109434]

35. Yuan M, Zhu H, Xu J, Zheng Y, Cao X, Liu Q. Tumor-Derived CXCL1 Promotes Lung Cancer Growth via Recruitment of Tumor-Associated Neutrophils. J Immunol Res. 2016; 2016:6530410. [PubMed: 27446967]

36. Yu PF, Huang Y, Han YY, Lin LY, Sun WH, Rabson AB, et al. TNFalpha-activated mesenchymal stromal cells promote breast cancer metastasis by recruiting CXCR2+ neutrophils. Oncogene. 2017; 36:482-90. [PubMed: 27375023]

37. Steele CW, Karim SA, Leach JD, Bailey P, Upstill-Goddard R, Rishi L, et al. CXCR2 Inhibition Profoundly Suppresses Metastases and Augments Immunotherapy in Pancreatic Ductal Adenocarcinoma. Cancer Cell. 2016; 29:832-45. [PubMed: 27265504]

38. Asfaha S, Dubeykovskiy AN, Tomita H, Yang X, Stokes S, Shibata W, et al. Mice that express human interleukin- 8 have increased mobilization of immature myeloid cells, which exacerbates inflammation and accelerates colon carcinogenesis. Gastroenterology. 2013; 144:155-66. [PubMed: 23041326]

39. Hoggatt J, Pelus LM. Hematopoietic stem cell mobilization with agents other than G-CSF. Methods Mol Biol. 2012; 904:49-67. [PubMed: 22890921]

40. Pelus LM, Fukuda S. Peripheral blood stem cell mobilization: the CXCR2 ligand GRObeta rapidly mobilizes hematopoietic stem cells with enhanced engraftment properties. Exp Hematol. 2006; 34:1010-20. [PubMed: 16863907]

41. Cui TX, Kryczek I, Zhao L, Zhao E, Kuick R, Roh MH, et al. Myeloid-derived suppressor cells enhance stemness of cancer cells by inducing microRNA101 and suppressing the corepressor CtBP2. Immunity. 2013; 39:611-21. [PubMed: 24012420]

42. Di Mitri D, Toso A, Chen JJ, Sarti M, Pinton S, Jost TR, et al. Tumour-infiltrating Gr-1+ myeloid cells antagonize senescence in cancer. Nature. 2014; 515:134-7. [PubMed: 25156255] 

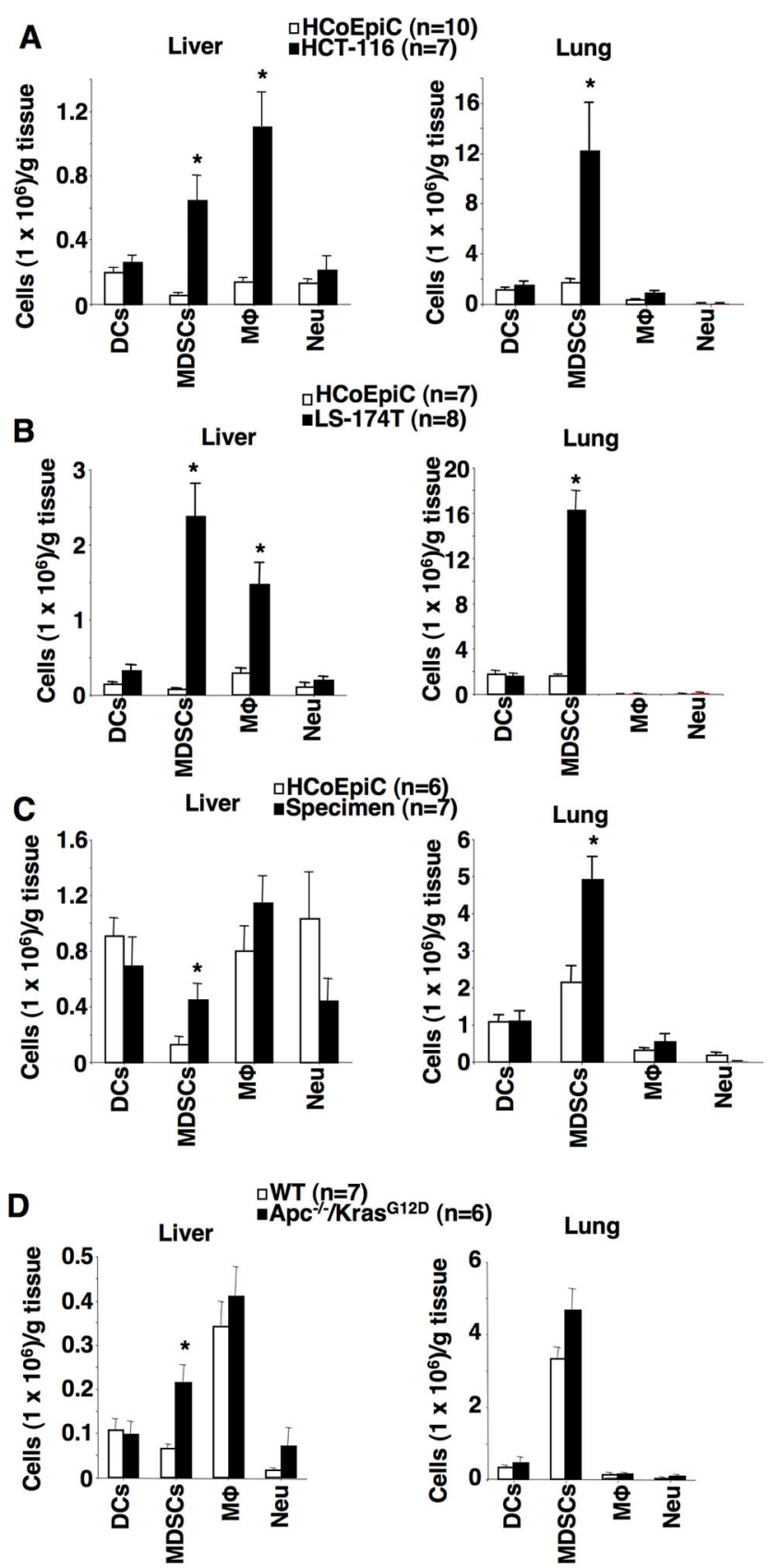

Fig. 1. Primary tumor induces an accumulation of gMDSCs in pre-metastatic liver (A-C) The numbers of DCs, gMDSCs, macrophages $(\mathrm{M} \phi)$, and neutrophils (Neu) in premetastatic livers and lungs of NSG mice injected with HCoEpiC cells, HCT-116 cells (A), LS-174T cells (B), or primary carcinoma cells isolated from human CRC specimen (C). (D) The numbers of DCs, gMDSCs, macrophages $(\mathrm{M} \phi)$, and neutrophils (Neu) in pre-metastatic livers and lungs taken from WT and $\mathrm{Apc}^{-/-/ K r a s}{ }^{G 12 D}$ mice. The error bar indicates \pm SEM. $* \mathrm{p}<0.05$. 
A

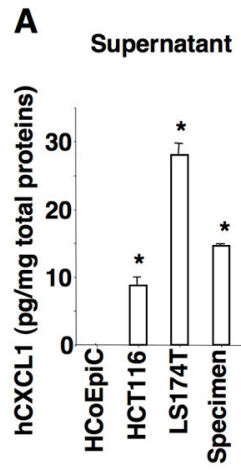

B

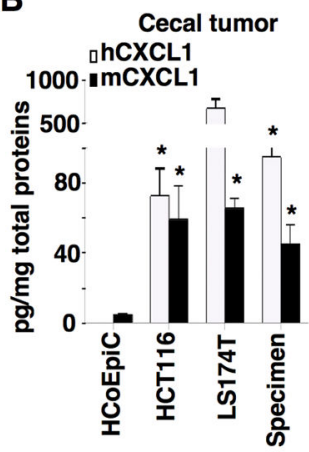

C

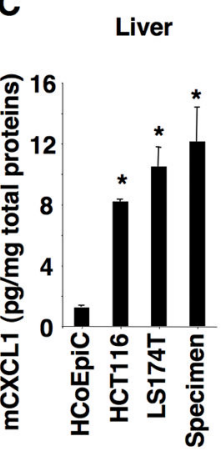

D

Blood

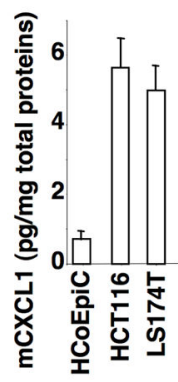

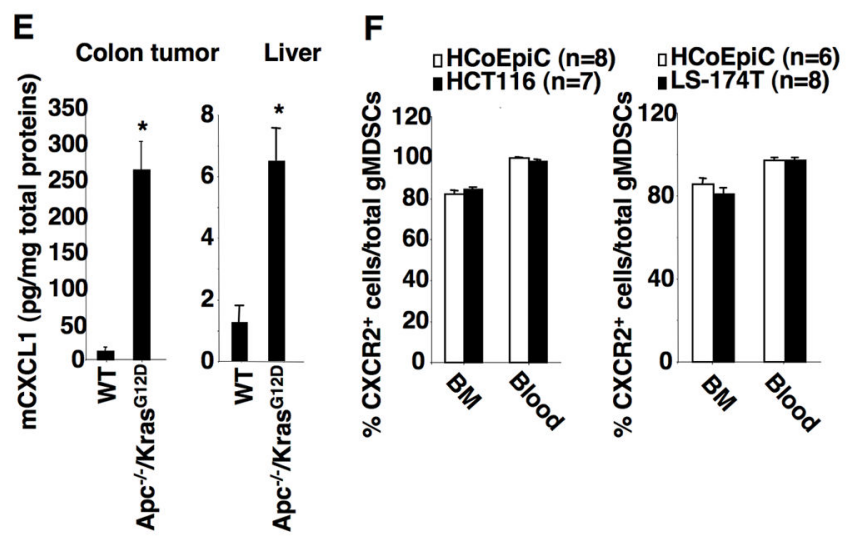

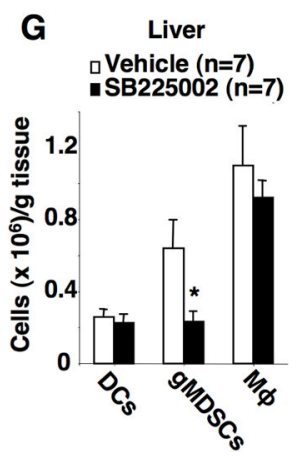

H
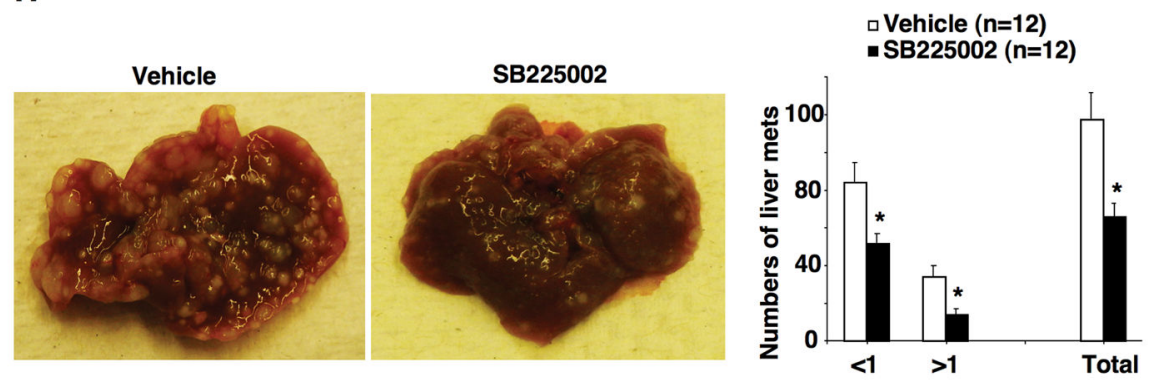

Fig. 2. The CXCL1-CXCR2 axis is required for recruitment of MDSCs into pre-metastatic liver and liver metastasis

(A) The protein levels of human CXCL1 in the cell culture supernatants of indicated cells.

(B-D) The protein levels of human and/or mouse CXCL1 in cecal tumor (B), pre-metastatic livers (C), and blood (D) of NSG mice injected with indicated cells as described in Fig. 1AC. (E) The protein levels of mouse CXCL1 in normal colonic tissues and liver of WT mice and colonic tumors and liver of $A \mathrm{pc}^{-/-} / \mathrm{Kras}^{G 12 D}$ mice. (F) Data represents the percentage of CXCR2-positive gMDSCs in total gMDSCs from bone marrow (BM) and peripheral blood taken from NSG mice injected with HCoEpiC cells, HCT-116 cells (left panel), or LS-174T cells (right panel) as described above. (G) The numbers of DCs, gMDSCs, and macrophages $(\mathrm{M} \phi)$ in pre-metastatic livers of NSG mice treated with vehicle or SB225002 after cecal injection of HCT-116 cells. (H) Gross view of liver metastatic tumors (left panels) and the average numbers of liver metastatic tumors at different size and total that includes all sizes 
(right panel) in NSG mice treated with vehicle or SB225002 after cecal injection of HCT-116 cells. The error bar indicates \pm SEM. * $p<0.05$. 
A
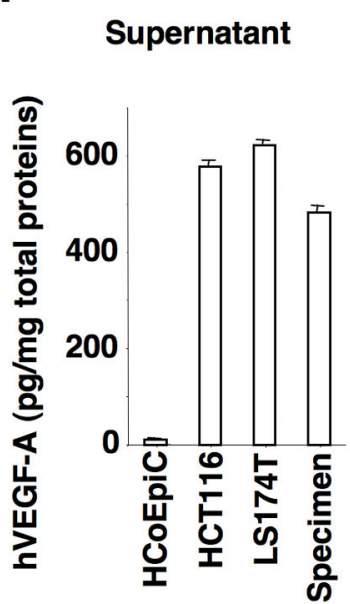

B

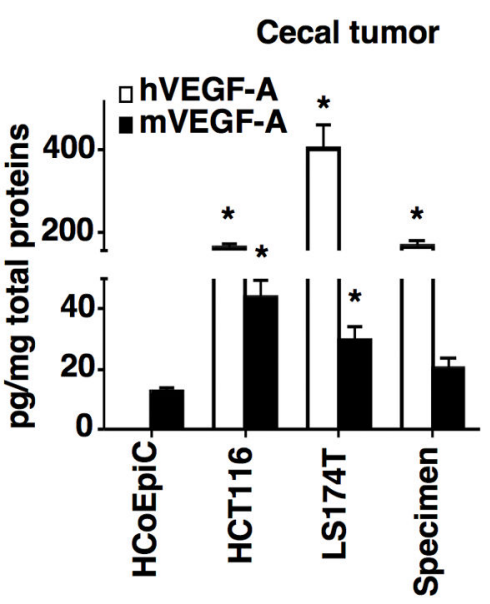

C

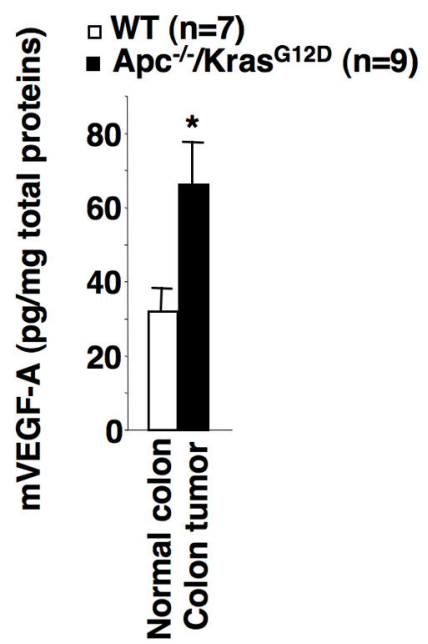

D

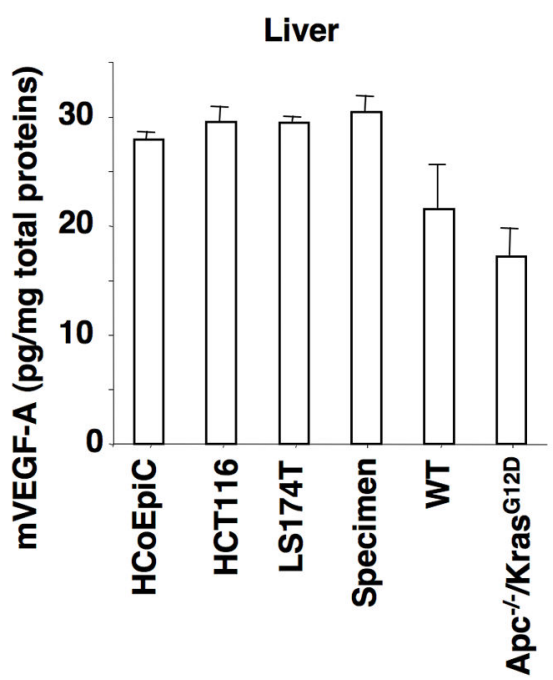

Fig. 3. VEGF-A is elevated in cecal tumors of NSG mice

(A) The protein levels of human VEGF-A in the cell culture supernatants of indicated cells.

(B) The protein levels of human and mouse VEGF-A in cecal tumors of NSG mice injected with indicated cells as described in Fig. 1A-C. (C-D) The protein levels of mouse VEGF-A in normal colonic tissues, colonic tumors, and pre-metastatic livers of WT and $\mathrm{Apc}^{-/-}$ $\operatorname{Kras}^{G 12 D}$ mice as well as in pre-metastatic livers of NSG mice injected with indicated cells as described in panel B. The error bar indicates \pm SEM. $* \mathrm{p}<0.05$. 
A

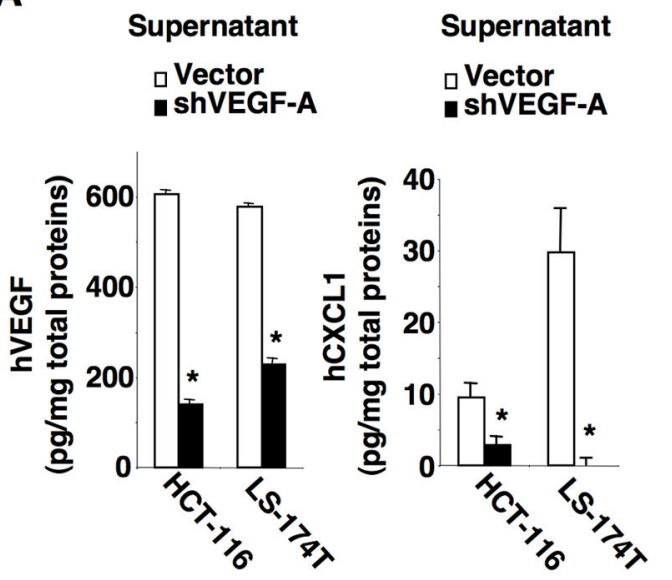

C

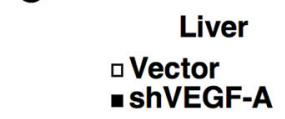

D
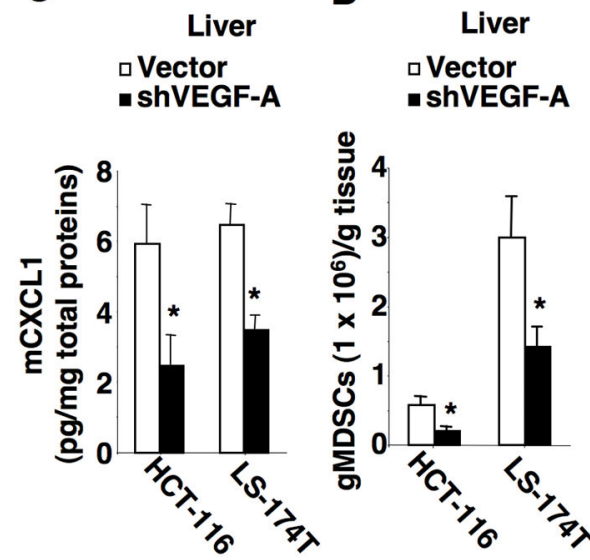

E
B
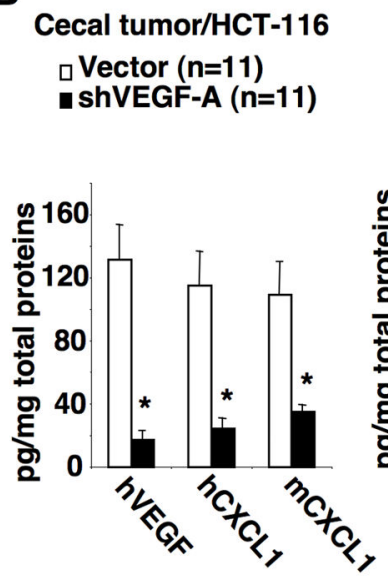

$\square$ Vector $(n=9)$

- shVEGF-A $(n=7)$
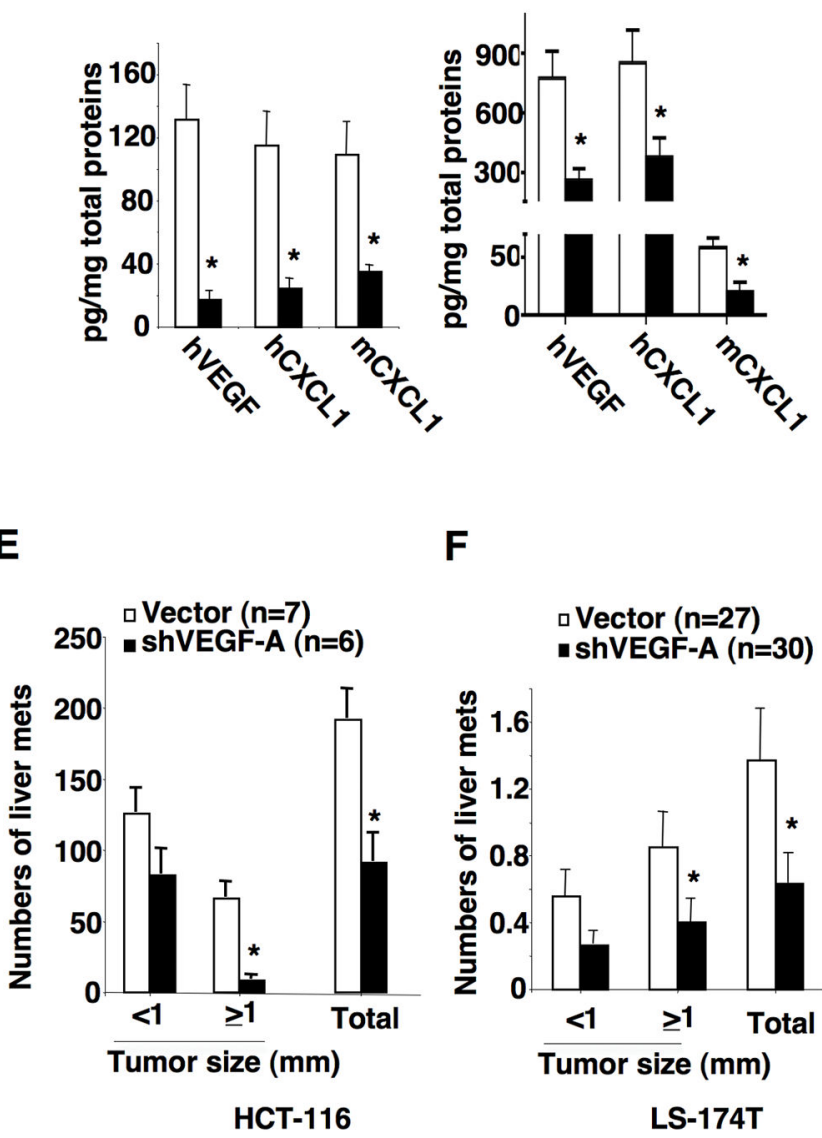

$\mathbf{F}$

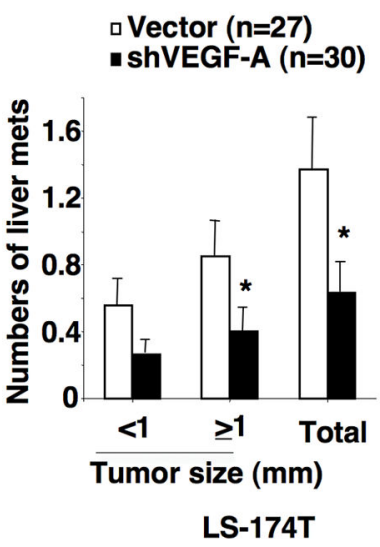

Fig. 4. VEGF is required for induction of CXCL1, infiltration of MDSC in pre-metastatic liver, and promotion of liver metastasis

(A) The protein levels of human VEGF-A (left panel) and CXCL1 (right panel) in the cell culture supernatants of indicated cells. (B) The protein levels of human VEGF-A, human CXCL1, and mouse CXCL1 in cecal tumors of NSG mice injected with HCT-116/vector or HCT-116/shVEGF-A (left panel), as well as LS-174T/vector or LS-174T/shVEGF-A cells (right panel) without developing liver metastasis. (C) The protein levels of mouse CXCL1 in pre-metastatic livers of NSG mice injected with indicated cells as described in panel B. (D) The numbers of gMDSCs in pre-metastatic livers of NSG mice injected with indicated cells as described in panel B. ( $\mathbf{E}-\mathbf{F})$ The average numbers of liver metastatic tumors at different size and total that includes all sizes in NSG mice injected with indicated cells as described in panel B. The error bar indicates \pm SEM. $* \mathrm{p}<0.05$. 

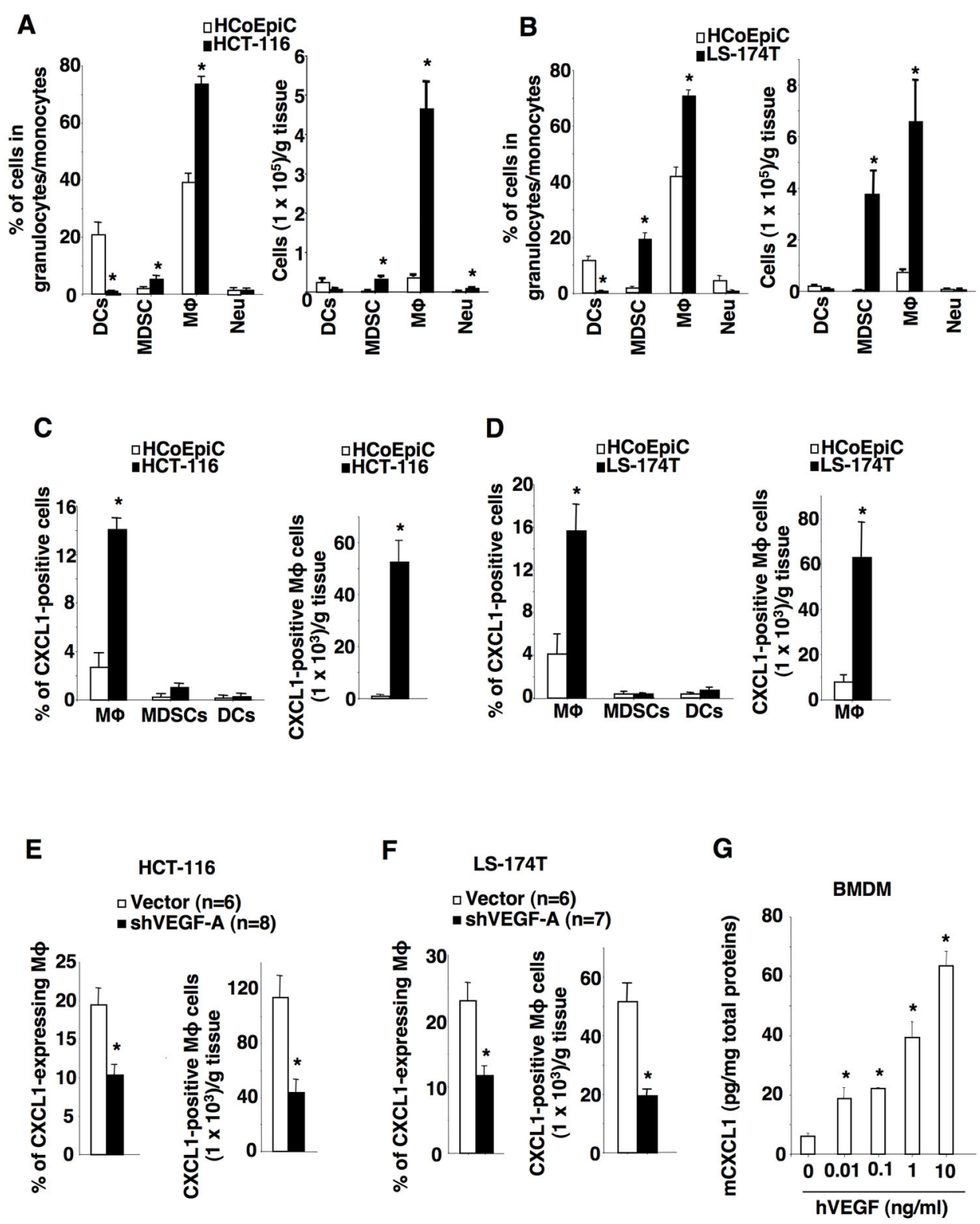

Fig. 5. Tumor cell-secreted VEGF stimulates tumor-associated macrophages to produce CXCL1 (A-B) The percentage (left panes) and numbers (right panel) of DCs, gMDSCs, macrophages $(\mathrm{M} \phi)$, and neutrophils (Neu) in normal cecum of NSG mice injected with HCoEpiC cells and cecal tumors of NSG mice without developing metastasis after injection of HCT-116 cells (A) or LS-174T cells (B). (C) Left panel, data represents the percentage of $\mathrm{CXCL1}^{+}$macrophages, $\mathrm{CXCL1}^{+}$gMDSCs, and CXCL1 ${ }^{+}$DCs in total macrophages, gMDSCs, and DCs in normal cecum and cecal tumors of NSG mice as described in panel A. Right panel, the numbers of $\mathrm{CXCL1}^{+}$macrophages in normal cecum and cecal tumors of NSG mice as described in panel A. (D) Left panel, data represents the percentage of $\mathrm{CXCL1}^{+}$macrophages, $\mathrm{CXCL1}{ }^{+}$gMDSCs, and $\mathrm{CXCL1}^{+}$DCs in total macrophages, gMDSCs, and DCs in normal cecum and cecal tumors of NSG mice as described in panel B. Right panel, the numbers of $\mathrm{CXCL1}^{+}$macrophages in normal cecum and cecal tumors of 
NSG mice as described in panel B. (E-F) Data represents the percentage of CXCL1 ${ }^{+}$ macrophages in total macrophages (left) and numbers of $\mathrm{CXCL1} 1^{+}$macrophages (right panel) in cecal tumors of NSG mice without developing metastasis after injection of HCT-116/ vector or HCT-116/shVEGF-A cells (E) as well as LS-174T/vector or LS-174T/shVEGF-A cells (F). (G) Protein levels of mouse CXCL1 in the supernatants of mouse bone marrowderived macrophages (BMDMs) treated with indicated dose of recombinant human VEGFA. The error bar indicates \pm SEM. $* \mathrm{p}<0.05$. 
A

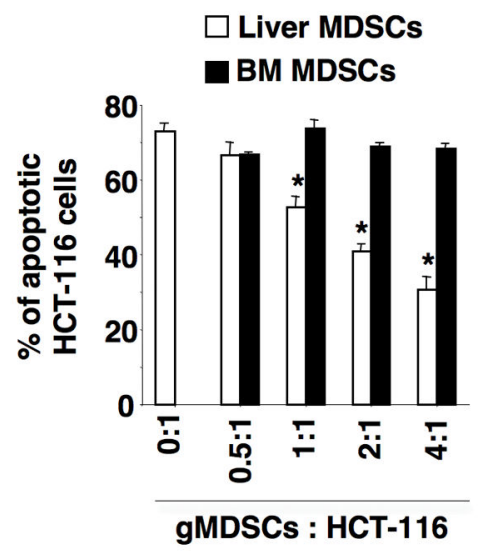

B

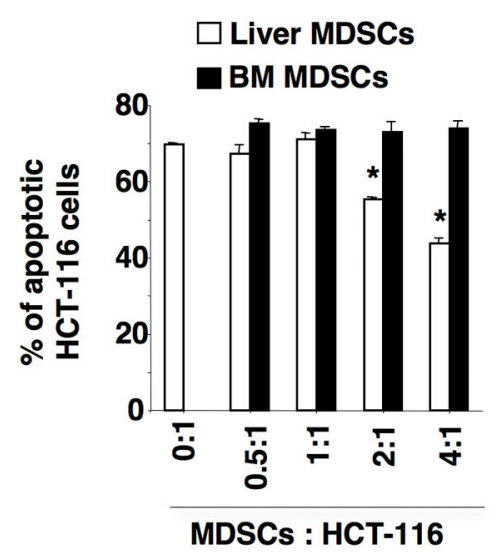

Fig. 6. MDSCs in pre-metastatic liver promote cancer cell survival

(A-B) The percentage of apoptotic HCT-116 cells after co-culture of HCT-116 cells with bone marrow-derived gMDSCs or gMDSCs isolated from pre-metastatic liver of NSG mice bearing HCT-116 tumor (A) or LS-174T tumor (B) at indicated ratio in transwell plates. The error bar indicates \pm SEM. $* \mathrm{p}<0.05$. 


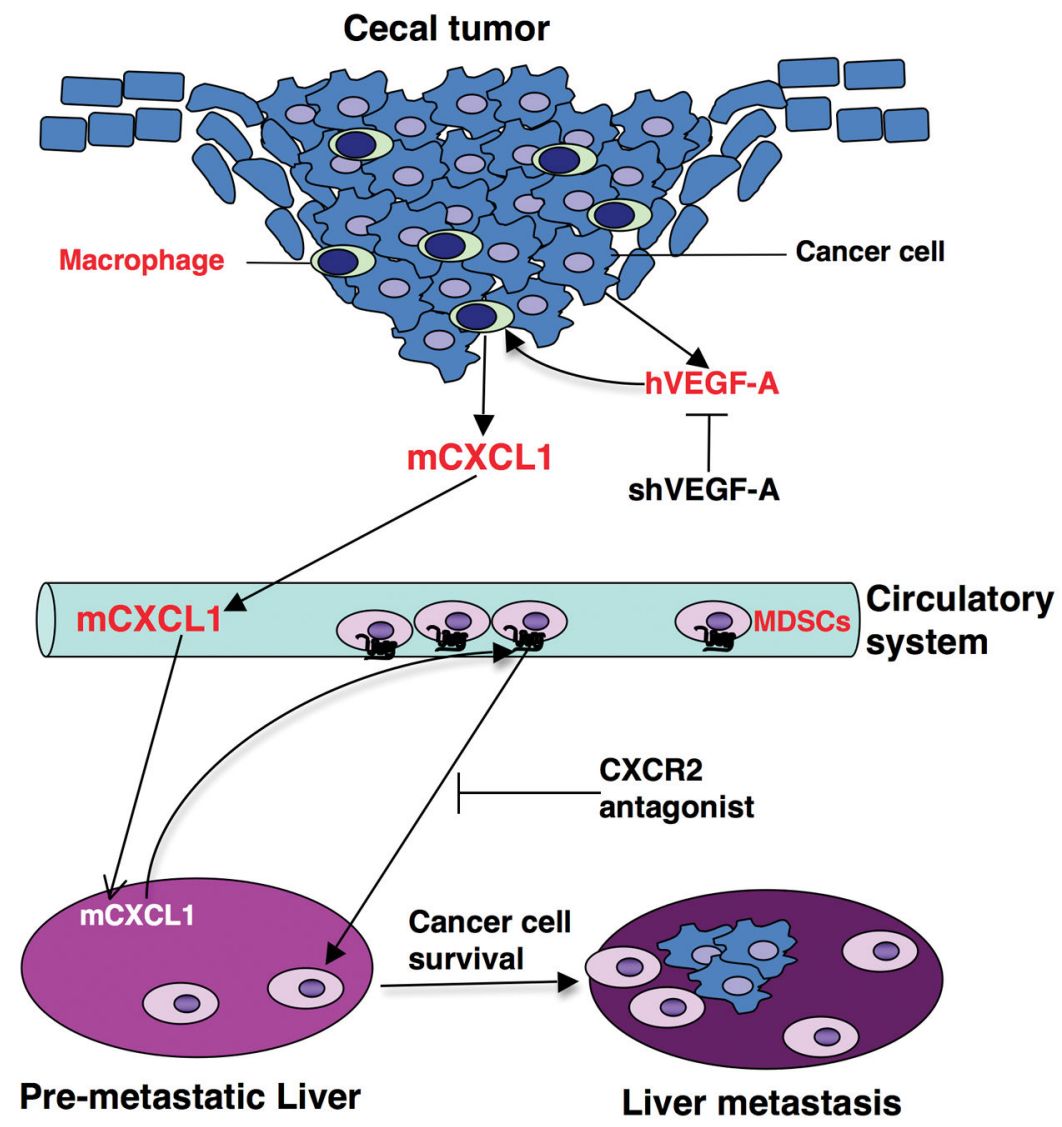

Fig. 7. A novel mechanism underlying the contribution of primary tumor to pre-metastatic niche formation and liver metastasis

Primary malignant cell-secreted VEGF-A stimulates primary tumor-associated macrophages to produce CXCL1 that recruits CXCR2-positive MDSCs from circulatory system into premetastatic liver. MDSCs in pre-metastatic liver promote liver metastases via induction of cancer cell survival in a mouse orthotopic model of CRC. 